\title{
Continuous-flow techniques for tests in clinical chemistry
}

\author{
D. W. NEILL AND J. R. DOGGART \\ From the Biochemistry Laboratory, Royal Victoria Hospital, Belfast, N. Ireland
}

The title of this paper is, for the hospital biochemist, synonymous with AutoAnalyzer techniques, and it is with the development and evolution of the AutoAnalyzer that it must inevitably deal. The next paper gives an account of the intensive developments which are taking place in the application of discrete analysis systems. This is perhaps the more difficult task, since, while there are many examples of this type there is effectively only one continuous-flow analytical technique.

To the industrial analytical chemist coming into the field of clinical chemistry 18 years ago, it seemed that the methods employed lacked the absolute character which obtain in the industrial field. The reasons implicit in the study of biological material soon became obvious and the work load overwhelming, so that any naive concept of bringing the cold purity of absolute analytical accuracy to bear in this new field was gladly abandoned. It is important, however, to remember this feeling, because there is a tendency to approach automated techniques in a highly critical fashion, examining the performance of this or that system against the yardstick of a sanctified manual system of clinical chemistry which never had any reality.

The principles of the single-channel AutoAnalyzer are now widely known, since subsequent changes in design have not fundamentally altered the approach so brilliantly described by Skeggs (1957). It was his recognition of the non-absolute character of clinical chemical methods, to which attention has been drawn above, which is fundamental to the success of the AutoAnalyzer. It was not important or even desirable to produce a machine capable of estimating urea concentration with absolute accuracy. If the machine could be guaranteed to treat every sample in exactly the same way so that standards were treated in the same way as tests, then the approach usual in manual methods would be operative. Indeed since machines have no free will and do not tire, it would be reasonable to expect greater precision.
A consideration of the flow diagram of a simple AutoAnalyzer determination is sufficient to define the concept of continuous-flow analysis. It is essential, if continuous-flow principles are to be used for separate samples, that samples be introduced sequentially and uniformly into the flowing stream of reagent. The sampler achieves this by the mechanical Geneva wheel type of mechanism and is also able to introduce water between samples so as to minimize the effects of carryover of sample from one cup to the next.

The movement of the liquids, both samples and reagents, is achieved by the action of the roller-bar peristaltic pump. Each flow tube is compressed by the pump at the same time so that in theory flow is uniform and simultaneous in all tubes. Smythe, Shamos, Morgenstern, and Skeggs (1967) have latterly drawn attention to the condition obtaining as each roller, in its cycle, lifts up off the platen, when a situation occurs whereby the wide-bore tubes are still occluded whereas the narrow-bore tubes are free, and non-simultaneous pumping is occurring. As will be shown, this is corrected in the newer type of AutoAnalyzer equipment, but it is clearly a point at which the simple AutoAnalyzer departs from the basic concept of all samples being treated alike.

The diluted, flowing serum samples are next deproteinized by continuous dialysis. It is this facility of ready deproteinization which is one of the major benefits of continuous-flow analysis and gives to it the flexibility which to some extent accounts for its ready application in the clinical chemical field. The concept is to some extent imperfect since the standards on which the calibration is based are generally aqueous and it is untrue that the rate of dialysis out of an aqueous stream is the same as that out of a stream rich in protein. Again, however, it must be remembered that, in manual analysis, calibration is usually against aqueous reference standards, so that no new principle is introduced. It is in the dialyser, however, that the first major 
contributions to sample interaction and nonuniformity of flow may arise, since the dialysed components, in progressively reducing concentration, enter an air-segmented stream whose segmentation does not necessarily correspond to that in the original sample stream.

The stream for analysis, whether the reagent stream containing the dialysate or the undialysed stream, is then pumped to the transducer. In the simplest estimation this is a colorimeter, but other transducers (flame photometer, fluorimeter, etc) can be used. At this stage it is essential to remove from the stream entering the transducer the segmenting air bubbles in order that the optical density or fluorescent emission may be read.

This is the second time that air segmentation has been mentioned and it has been held by many that this feature is the key to successful continuous-flow analysis. All reagent lines, after the initial mixing following the peristaltic pump, are segmented by air bubbles and these serve to separate the specimens, to assist in the tumbling action in the mixing coils, and, because of the slight positive pressure in the system, to 'sweep' the tubing walls. It is important that the bubble pattern be uniform if the liquid stream corresponding to a given sample is to be uniform in concentration along its length. This last is clearly the primary condition for obtaining a smooth peaking signal for recording. As has been said, the manufacturers now incorporate positive steps to guarantee uniform air-bubble segmentation, but this is not a feature of the basic AutoAnalyzer under consideration.

However, at the transducer the air-bubble segmentation is deliberately removed, so that its advantages, as outlined above, are lost. Thus sample interaction becomes possible and the effects of carryover, usually referred to here as 'poor wash', become evident.

The foregoing, necessarily compressed, examination of the simple continuous-flow analytical method has indicated some of the points at which the underlying concept of the system, $i e$, uniformity of operation for all specimens and absence of interaction, falls short of being realized in practice. This is, however, less important than it might otherwise appear because the recorder presentation, in the hands of an experienced operator, not only serves as the basis for reading the results, but also reveals, in the shape of the 'rise curve', 'fall curve', and peak, the presence of those sources of error to which I have drawn attention. All users are familiar with those peaks which are unfit for reporting, but it is only when one endeavours to program a computer to provide an error message, or when one is confronted by one of the newer discrete analysers that the value of the information conveyed by the standard AutoAnalyzer curve becomes apparent.

The manufacturers of the AutoAnalzyer refer to this information gleaned from the recorder chart by an experienced operator as 'machine function'. While it can hardly be regarded as clear-cut data regarding the function of the individual modules of the system it does go a long way towards warning of imperfect analyses and thus towards the idea of 'automation'.

It is hardly necessary to trace the evolution of the continuous-flow analyser through the stages of double-channel systems to multichannel systems. There can be little doubt as to the benefit of the so-called electrolyte system. Clinical requirements for the simultaneous estimation of $\mathrm{Na}, \mathrm{K}, \mathrm{Cl}$, and $\mathrm{CO}_{2}$ became increasingly pressing, and were met by the linking of four single channels side by side and the development of a flame photometer capable of continuous working. The performance of this particular block of tests is clinically justifiable, to the extent that it is usual to regard them as inseparable. It was then technically a simple matter to add a fifth channel, estimating urea, since the use of this block of tests was often related to studies of renal disease, and in most cases urea estimation was requested as well as electrolytes.

This five-channel analyser could thus be seen as a functional piece of equipment designed for a clearly defined purpose. The next development is, however, philosophical in its nature. Having a five-channel system capable of producing five results simultaneously on a sample of serum, is there any practical advantage in having a separate analytical system, manual or automatic, for carrying out any one of the five estimations? If the estimation of urea is requested for a particular sample, it is more efficient to act as if all five analyses had been requested. The clinician can ignore the information he has not requested but may even find it of value. This philosophy has informed all recent developments in the use of continuous-flow analysis in the clinical chemical field, stimulated in large part by the manufacturer. It involves obtaining as many estimations as possible on each patient specimen submitted, and it leads almost inevitably to the concept of screening. A consideration of the dialectic for and against screening is outside the scope of this paper, but it has been the reason for the particular developments which have taken place in the production of commercial continuous-flow systems. A number of centres in the United Kingdom are using combinations of single-channel AutoAnalyzer systems to obtain a number of results for each specimen submitted. Experience in the Royal Victoria Hospital has been with the so-called simultaneous multiple 
analyser, in which the single-channel AutoAnalyzer approach is considerably modified.

In the SMA-12, the system of continuous-flow analysis already described is employed, but the 12 flowing streams are so adjusted in length that the final solutions to be read by the transducers reach them in sequence. Each transducer is switched to transmit a signal while its own analytical stream is carrying test-derived component and the single recorded thus receives 12 signals, one after the other. Further, the parameters of the system are so adjusted that the 'rise' and 'fall' components of the signal are rapid in relation to the steady maximum and the recorder only records during a portion of the steady state. This recording thus consists of a short plateau line for each component, the plateaux being displaced horizontally from one another by the movement of the paper. While this is attractive in the sense that the record is no longer a peak requiring a peak height measurement, but can be drawn on preprinted scaled paper to give an immediately readable result, it can be seen that, since only a small section of the signal plateau is visible, all the inherent information available from the single AutoAnalyzer peak curve is lost and aberrant behaviour in the system is not always recognizable. Most important of course is the need to guarantee that the recorded plateau is in the centre of the steady state signal, $i e$, that the 'phasing' of the analytical streams is correct. Earlier forms of the SMA-12 incorporated a phasing recorder which sampled the whole signal from each channel and indicated where the plateau record was occurring. It was possible to recognize obviously bad signals also. The newer equipment, the SMA-12/60, is provided with an oscilloscope on which the actual changes of concentration with time are displayed continuously for each analytical channel. Thus the operator can have information on performance which is usually available only on the single-channel type recorder chart.

In discussing the performance of multichannel continuous-flow analysers, our data are only available for the earlier SMA-12 since the SMA-12/60 is only just becoming available in the UK. With this proviso Table I sets out the quality control figures for reference sera included in routine SMA-12 batches over 15 days, compared with similar data from a single-channel analyser on glucose estimations over one month. From this it will be seen that the multichannel results all show precision within the generally accepted limits. Turning to the single-channel results the precision is clearly even better. It is our general experience that while the SMA-12-type analyser is satisfactory for this type
TABLE I

PRECISION IN CONTINUOUS-FLOW SYSTEMS

\begin{tabular}{|c|c|c|}
\hline Estimation & Mean & $S D$ \\
\hline \multicolumn{3}{|l|}{ SMA-12 (66 specimens over two weeks) } \\
\hline $\mathrm{Na}(\mathrm{m}$-equiv/l) & $138 \cdot 56$ & $1 \cdot 38$ \\
\hline $\mathrm{K}(\mathrm{m}$-equiv/l) & 5.93 & $0 \cdot 12$ \\
\hline $\mathrm{Cl}(\mathrm{m}$-equiv/l) & $108 \cdot 15$ & $2 \cdot 25$ \\
\hline $\mathrm{CO}_{2}(\mathrm{~m}$-equiv/l) & $17-45$ & $0 \cdot 58$ \\
\hline Total protein $(\mathrm{g} / 100 \mathrm{ml})$ & $7 \cdot 69$ & $0 \cdot 11$ \\
\hline Albumin $(\mathrm{g} / 100 \mathrm{ml})$ & $2 \cdot 25$ & 0.05 \\
\hline $\mathrm{Ca}(\mathrm{mg} / 100 \mathrm{ml})$ & $13 \cdot 59$ & $0 \cdot 23$ \\
\hline Alkaline phosphatase (K-A units $/ 100 \mathrm{ml}$ ) & $16 \cdot 56$ & 0.78 \\
\hline Total bilirubin $(\mathrm{mg} / 100 \mathrm{ml})$ & $3 \cdot 37$ & $0 \cdot 23$ \\
\hline Blood urea $(\mathrm{mg} / 100 \mathrm{ml})$ & 143.90 & $2 \cdot 11$ \\
\hline Glucose $(\mathrm{mg} / 100 \mathrm{ml})$ & $188 \cdot 15$ & $8 \cdot 51$ \\
\hline SGOT (Karmen units) & $172 \cdot 27$ & $5 \cdot 13$ \\
\hline \multicolumn{3}{|l|}{ Single-channel AutoAnalyzer (one month) } \\
\hline Glucose $(251$ specimens $)(\mathrm{mg} / 100 \mathrm{ml})$ & $118 \cdot 7$ & $2 \cdot 4$ \\
\hline (40 specimens) $(\mathrm{mg} / 100 \mathrm{ml})$ & $234 \cdot 3$ & $3 \cdot 3$ \\
\hline
\end{tabular}

of work, its performance can be less stable over a long period of time than in the conventional singlechannel equipment.

\section{SUMMARY}

The continuous-flow type of clinical chemical analyser, both in its original form and in the more sophisticated, multichannel versions now becoming available, has performed more than adequately over more than a decade. It can indeed be claimed that it has transformed the practice of routine clinical chemistry, not least in the direction of improved precision. It has resulted too in the creation of a whole new corpus of methods as a result of the stimulus of attempting to use its undoubted ability to save labour in ever more diverse analytical problems. Perhaps more questionable is its creation of the concept of multichannel analysis, based on the thesis that any information is valuable. It is too convenient to regard this as synonymous with believing that this type of information is good because it can readily be obtained and that it is unnecessary to seek for other data less easily obtained. If the development of other systems of mass production analysis make it possible to undertake investigations as yet untried, then the present dependence on the AutoAnalyzer will be replaced by an increased versatility which is becoming increasingly desirable.

\section{REFERENCES}

Skeggs, L. T. (1957). An automatic method for colometric analysis, Amer. J. clin. Path., 28, 311-322.

Smythe, W. J., Shamos, M. H., Morgenstern, S., and Skeggs, L. T. (1967). SMA-12/60: a new sequential multiple analysis instrument, in Automation in Analytical Chemistry, Volume 1, p. 105-113. 